\title{
ASPECTOS BÁSICOS SOBRE LA EDUCACIÓN EN LÍNEA Y LA COBERTURA DE INTERNET EN LAS ZONAS DE INFLUENCIA DEL INSTITUTO TECNOLÓLIGO SUPERIOR ALMIRANTE ILLINGWORTH.
}

Autor.

Rolando J. Alvarez

Docente titular Instituto Tecnológico Illingworth

rolandoj@aitec.edu.ec

\author{
Coautor. \\ Dávila F. Moya \\ Docente titular Instituto Tecnológico \\ Illingworth \\ davilaf@aitec.edu.ec
}

\author{
Coautor. \\ Lázaro R.Cruz \\ Docente titular Instituto Tecnológico \\ Illingworth \\ Ireigosa@aitec.edu.ec
}

País Origen: Ecuador

Palabras claves: Desarrollo, educación, pedagogía y sociedad

Keywords: Development, education, education and society
Recibido: 11 de Marzo del 2016

Aceptado: 15 de Abril del 2016

\section{SUMMARY}

$\mathrm{n}$ the development of this article, an analysis is made that both the classroom and blended learning mode have remained along these years, and other modalities has changed both their nomenclature as its conceptualization.

Thus, the current regulation makes a distinction between the online mode, which organizes educational interaction of teacher and student, in real time and deferred and distance learning, which for synchronous development is fundamental tutorial work and administrative and organizational support centers.

\section{RESUMEN}

n el desarrollo del presente artículo se realiza un análisis que tanto la modalidad presencial y semipresencial se han mantenido a lo largo de estos años, y el resto de modalidades ha ido cambiando tanto su nomenclatura como su conceptualización. Así, el reglamento actual hace una diferenciación entre la modalidad en línea, que organiza la interacción educativa del profesor y el estudiante, en tiempo real y diferido y la modalidad a distancia, que para su desarrollo es fundamental la labor tutorial sincrónica y el respaldo administrativo-organizativo de centros de apoyo. 
a conceptualización de las modalidades de estudio en el Ecuador han venido evolucionando en los últimos años, siendo así que en Enero de 2009, el CONESUP aprueba el Reglamento Codificado de Régimen Académico del Sistema Nacional de Educación Superior, e incluye en su artículo 6 la oferta de modalidades para las diferentes carreras: presencial, semi presencial y a distancia.

En el año 2002, cuando el CONESUP aprueba el Reglamento General de los Institutos Superiores Técnicos y Tecnológicos del Ecuador, donde en su artículo 82 establecía que:

"La Actividad docente para la formación de pregrado en los niveles técnico, tecnológico y equivalentes, podrá ofrecerse a través de las modalidades presencial, semipresencial y virtual, siempre que cumplan con las normas establecidas por el Reglamento de Régimen Académico del Sistema Nacional de Educación Superior aprobado por el CONESUP".

Para los fines legales pertinentes, en todos los casos, las modalidades semipresencial y virtual se consideraran a distancia.

El actual Reglamento de Régimen Académico del Consejo de Educación Superior, aprobado y codificado en Septiembre de 2015,en su artículo 40, amplia las modalidades de estudio o aprendizaje a las siguientes:
a) Presencial
b) Semipresencial
c) Dual
d) En línea; $y$
e) A distancia,

Se observa que tanto la modalidad presencial y semipresencial se han mantenido a lo largo de estos años, y el resto de modalidades ha ido cambiando tanto su nomenclatura como su conceptualización. Así, el reglamento actual hace una diferenciación entre la modalidad en línea, que organiza la interacción educativa del profesor y el estudiante, en tiempo real y diferido y la modalidad a distancia, que para su desarrollo es fundamental la labor tutorial sincrónica y el respaldo administrativo-organizativo de centros de apoyo.

Por otra parte, el Instituto Tecnológico Superior Almirante Illingworth que en una primera etapa funcionaba bajo la modalidad presencial, fue evolucionando hacia la modalidad semipresencial, en la que se encuentran matriculados el $95 \%$ de sus estudiantes, procedentes de zonas geográficas de toda la provincia, en su mayoría trabajadores activos, por lo que se hace necesario comenzar una planificación estrategia para incursionar en la modalidad en línea, de forma que se pueda dar respuesta a la demanda de formación en toda la provincia del Guayas. Es de señalar que la educación en línea está llamada a dar respuesta a personas que ya por su ubicación geográfica o por aspectos laborales les resulta difícil asistir a una Institución de forma presencial, además que por estar basada en la tecnología, da paso al aprendizaje sin limitaciones de lugar, tiempo, ocupación o edad de los estudiantes

\section{DESARROLLO}

\section{ASPECTOS TEÓRICOS METODOLÓGICOS SOBRE LA EDUCACIÓN EN LÍNEA}

El Internet acabará produciendo cambios sustanciales en los Sistemas de Educación de todos los países y en particular en el Sistema de Educación Superior, como ya ha ocurrido en otros sectores como la comunicación, la industria, los servicios etc, propiciando así nuevos modelos de aprendizaje, entre ellos la modalidad en línea.

Conceptualmente se entiende por educación el línea, en algunas ocasiones denominada e-learning o educación virtual) a aquella en la que los docentes y los estudiantes participan en un entorno digital mediante redes de computadoras, haciendo uso intensivo de las facilidades que proporciona el internet y las tecnologías digitales.

Otros autores consideran que la educación en línea es una modalidad con características propias que se diferencia tanto de la educación a distancia como de la educación presencial. 
Una de las características fundamentales de la llamada educación en línea, es que se implementa usualmente a través de plataformas digitales para la gestión de cursos, conocidas como Learning Management System, que para su realización requiere que los estudiantes colaboren entre sí , trabajando de forma conjunta, generando la colaboración que se requiere de los estudiantes, sin que medie encuentros presenciales, con la implementación de encuentros sincrónicos y asincrónicos que se producen a través de las plataformas establecidas.

En general, mientras la educación a distancia se apoya principalmente en la distribución de materiales, físicos y/o electrónicos, en la educación en línea son fundamentales las actividades que los estudiantes deben resolver para aprender.

El Reglamento para carreras y programas académicos en modalidades en línea, a distancia y semipresencial o convergencia de medios, define en su art 4 y 5 las modalidades que se muestran en la tabla 1.

\section{EDUCACIÓN A DISTANCIA}

El componente de docencia, el de prácticas de aplicación y experimentación de los aprendizajes y el de aprendizaje autónomo, están mediados por el uso de tecnologías y entornos virtuales bajo plataformas de interacción y por la articulación de múltiples recursos didácticos (físicos y digitales). Para su desarrollo, es fundamental la labor docente y técnica docente con la tutoría sincrónica y asincrónica, y el respaldo administrativo-organizativo de centros de apoyo.

\section{EDUCACIÓN EN LÍNEA O VIRTUAL}

El componente de docencia , el de prácticas de aplicación y experimentación de los aprendizajes, y el aprendizaje autónomo están mediados fundamentalmente por el uso de las tecnologías interactivas multimedia y entornos virtuales que organizan la interacción educativa de los actores del proceso educativo, en tiempo real o diferido a través de plataformas informáticas académicas, que facultan la labor tutorial sincrónica y asincrónica

En general, se consideran rasgos distintivos de la modalidad en línea los siguientes:

- $\quad$ Profesores y estudiantes interactúan en un entorno digital durante el proceso de aprendizaje

- El uso pedagógico de las Tecnologías de la Información y la comunicación y la aplicación de la web 2.0 a la educación

- $\quad$ Trabajo colaborativo sincrónico y asincrónico entre los estudiantes a partir de actividades planificadas.

\section{SITUACIÓN DE LA COBERTURA DE INTERNET}

Una vez establecidos los principales aspectos teóricos metodológicos de la educación en línea o virtual, se indagó cómo se encuentra actualmente la cobertura de internet, tanto en la provincia del Guayas como en el país.

Según el portal de Ecuador en cifras del Instituto Nacional de Estadísticas y Censos en su publicación acerca de las tecnologías de la información y comunicaciones (TIC's) 2013, de la Agencia de Regulación y control de las Telecomunicaciones y del Ministerio de Telecomunicaciones y Sociedad de la Información, (Ver Anexos) se puede constatar que:

a) El acceso a Internet en hogares presenta una clara tendencia en ascenso que va desde un 11,8 $\%$ en el 2010 a $28,3 \%$ en el 2013, y a tener una densidad de internet en Junio de 2015 de $40,71 \%$, de la población a nivel nacional y un 58,34\% a nivel de la provincia del Guayas. (Densidad de Internet se refiere a cantidad de abonados por cada 100 habitantes).

c) En cuanto al acceso a Internet desde cybers encontramos que la provincia del Guayas cuenta con 516 establecimientos, distribuidos en todos los cantones de la provincia, siendo el cantón Guayaquil la de mayor número con 390. (Información 20 de Junio 2015)

d) Dentro de los objetivos del Plan Nacional de Desarrollo de Banda Ancha del Ministerio de Telecomunicaciones y Sociedad de la Información, se considera los siguientes:

* Mejorar la calidad de vida de los ecuatorianos mediante el uso, introducción y apropiación de las nuevas tecnologías de la información y la comunicación. 
* Decremento de los precios de acceso al servicio de internet de banda ancha.

* Mejorar la calidad de vida de los ecuatorianos mediante el uso, introducción y apropiación de las nuevas tecnologías de la información y la comunicación.

* Decremento de los precios de acceso al servicio de internet de banda ancha.

* Permitir a todos los ecuatorianos independientemente de su condición socioeconómica y ubicación geográfica en los accesos de banda ancha con calidad y calidez.

e) La Muy llustre Municipalidad de Guayaquil, comenzo con el plan "Guayaquil ciudad digital" en el cual se instalarán 6000 puntos de internet gratuitos wifi en varias zonas de la ciudad, siendo el Instituto uno de los beneficiarios de este plan que ya está al servicio de los estudiantes.

\section{SISTEMAS DE GESTION DE APRENDIZAJE O LEARNING MANAGEMENT SYSTEM}

Un sistema de gestión de aprendizaje es un software que funciona en un servidor web para la administración, distribución y controles de las actividades académicas no presenciales de una institución, permitiendo realizar un trabajo asíncrono entre los estudiantes y docentes.

Un sistema de gestión de aprendizaje debería realizar las siguientes funciones fundamentales:

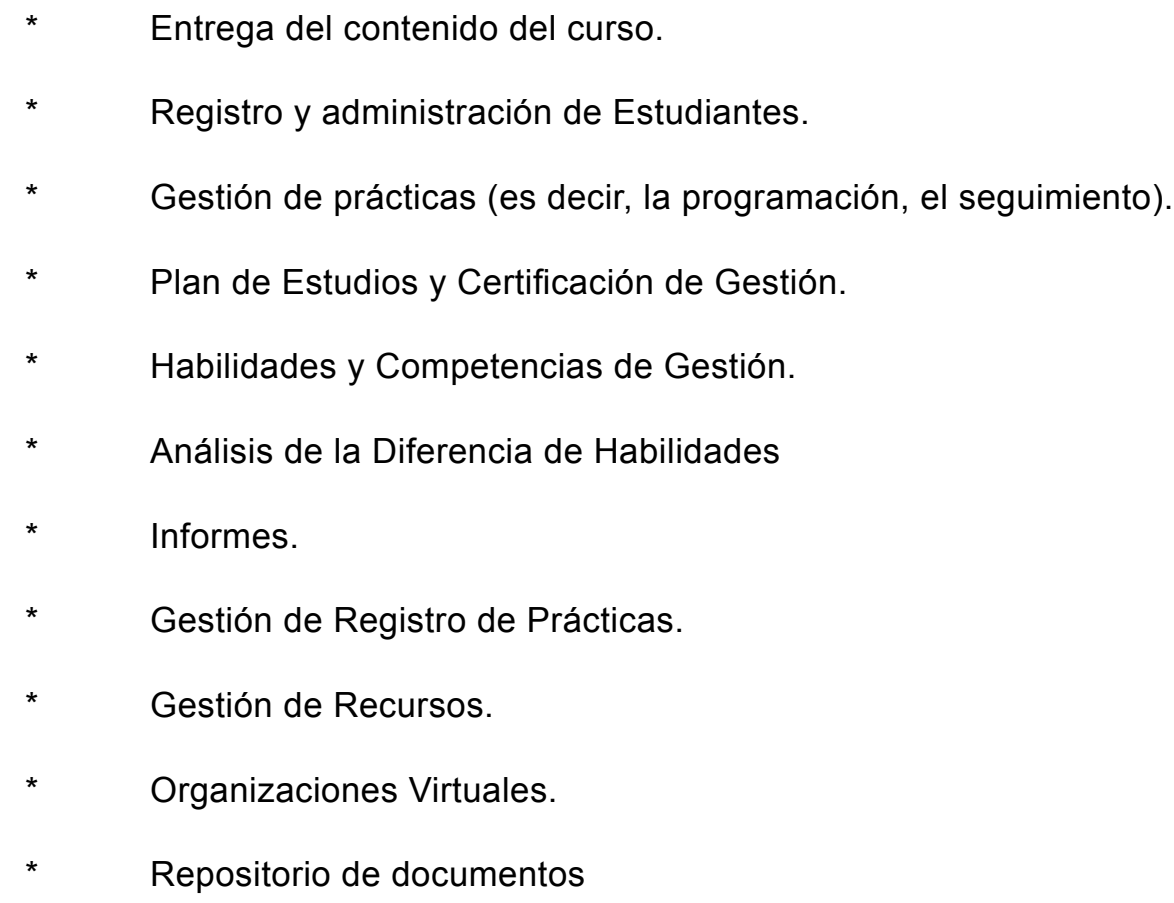

Actualmente existen varios sistemas de gestión de aprendizaje, que los podemos clasificar según se nuestra en la tabla 2:

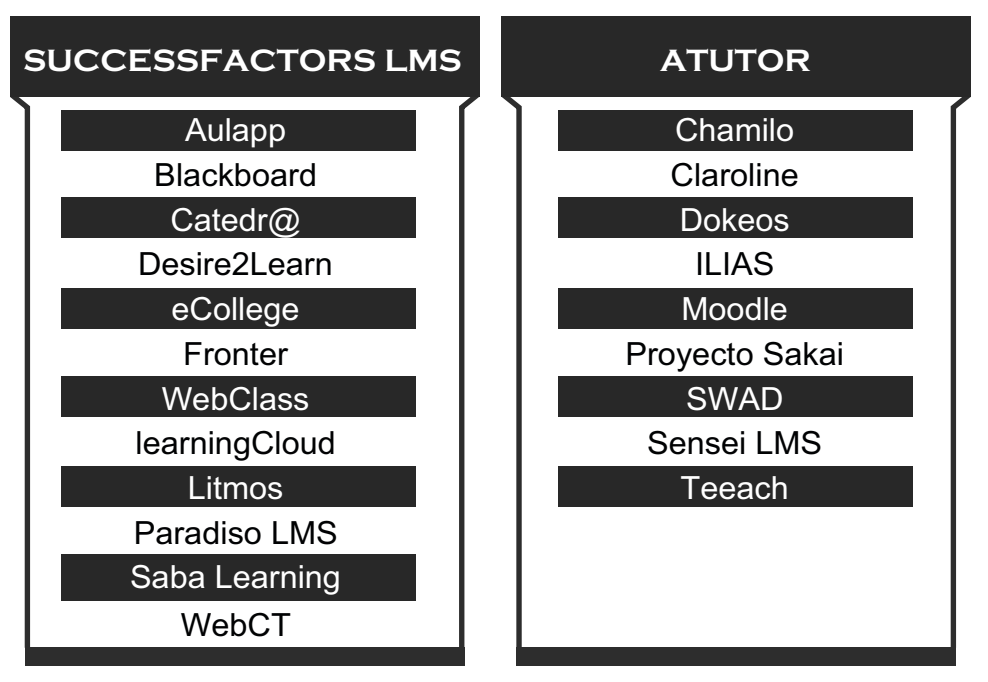


Entre todos los sistemas anteriores se destaca Moodle por ser un software intuitivo, de fácil aprendizaje compatible con la mayoría de sistemas operativos y además gratuito. Su modelo se muestra en la figura 1

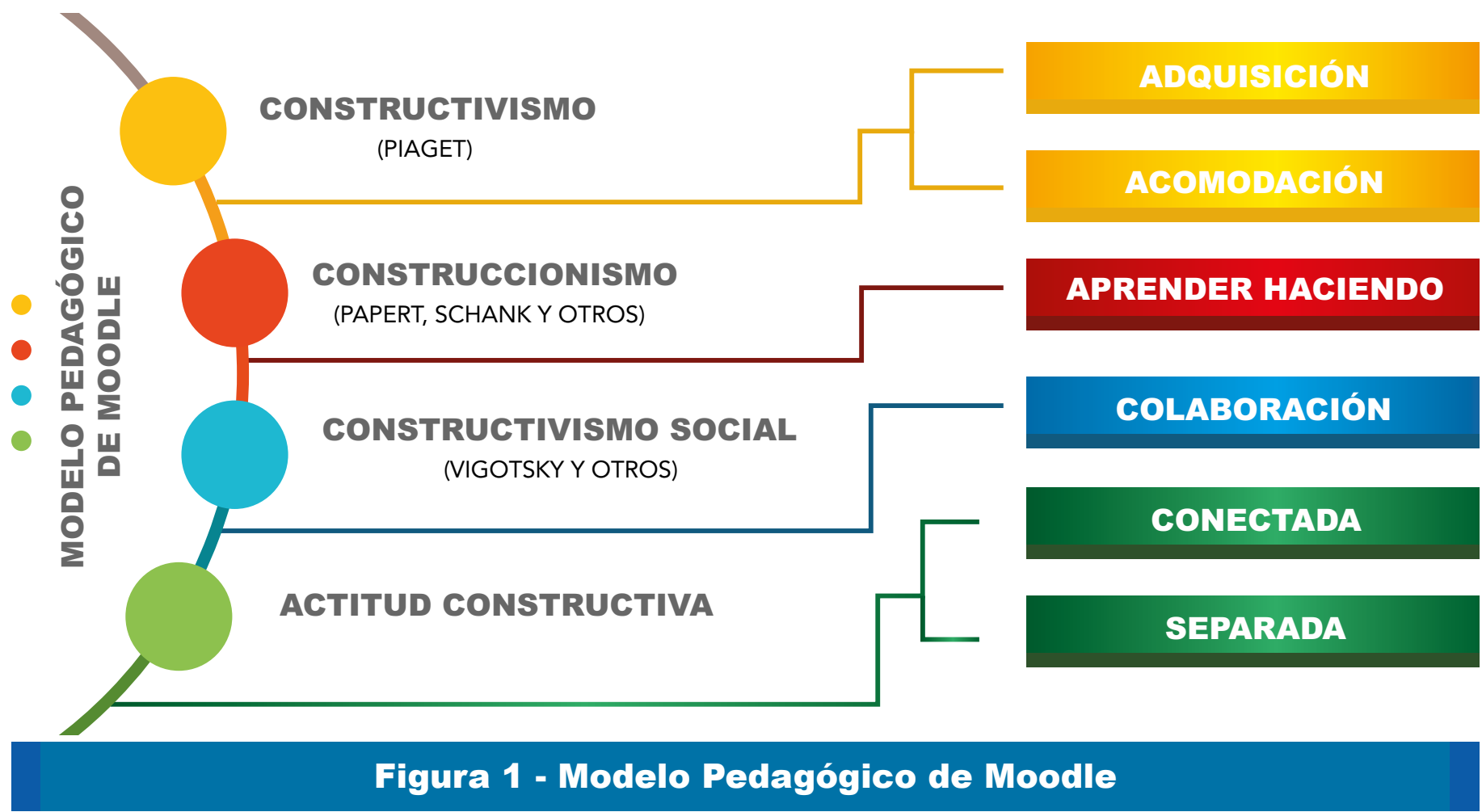

El modelo está basado en la pedagogía construccionista social donde el estudiante construye su conocimiento a base de las herramientas e individuos que intervienen en su proceso haciéndolo también colaborativo. Por ello se puede decir que favorece el aprendizaje constructivo y colaborativo, pero además flexibiliza horarios y distancias, diversifica las fuentes de información y democratiza los agentes de conocimiento. Moodle promueve la comunicación, la participación, la colaboración y el feedback inmediato.

Resulta apropiado para clases en línea, a distancia, así como también para complementar y enriquecer el aprendizaje presencial. Permite realizar una interesante variedad de actividades individuales y colaborativas, subir e integrar contenidos bajo diferentes formatos entre otras posibilidades.

Moodle es el sistema de gestión de aprendizajes más usada a nivel mundial, siendo utilizada en universidades reconocidas y/o como parte del modelo académico en ciertos países, en modalidades como

$\begin{array}{ll}* & \text { Clases virtuales y conferencias } \\ * & \text { Portales institucionales } \\ * & \text { Campus Virtuales } \\ * & \text { Tutorías } \\ * & \text { Grupos de trabajo e investigación }\end{array}$

En Moodle se pueden realizar las actividades mostrada en la figura 2 


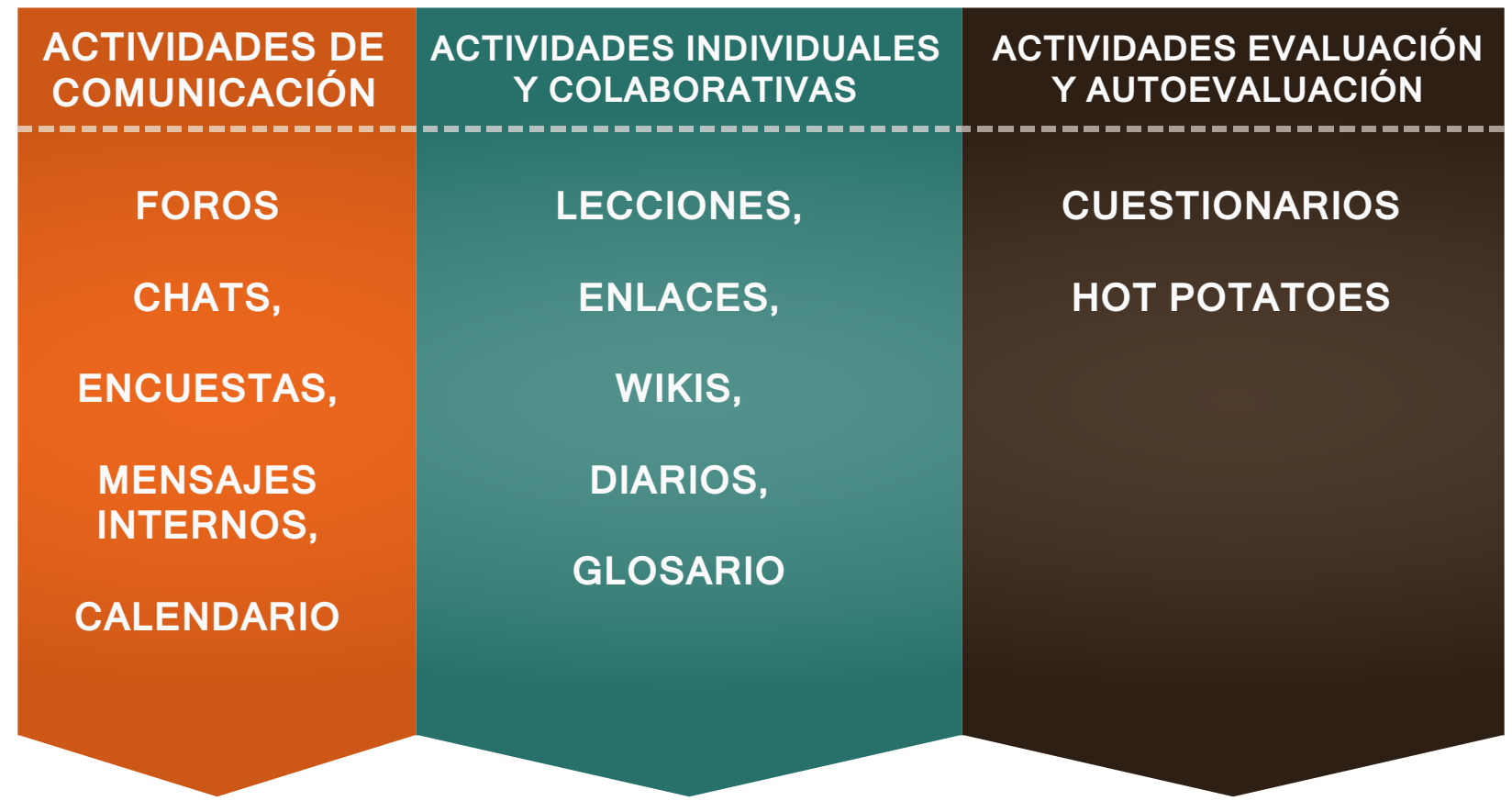

Figura 2 - Actividades de Moodle

\section{SITUACIÓN DE LA INFRAESTRUCTURA TECNOLÓGICA EN EL INSTITUTO}

Se considera que con la infraestructura tecnológica que el Instituto posee actualmente, estamos preparados para brindar el servicio requerido para la modalidad en línea, contando con la experiencia que hemos adquirido dando soporte a la modalidad semi presencial tanto para la comunicación en tiempo sincrónico como asincrónico.

Se cuenta con un sistema de gestión de aprendizaje utilizado mundialmente (MOODLE), que se ha utilizado hasta el momento para la comunicación docente-estudiante, y el trabajo colaborativo a través de chats, foros, tareas etc. Esta plataforma es OPEN SOURCE, o se de software libre o gratuito, lo que no representa costo de licencia para su utilización.

Además dispone hace un año de una plataforma de aulas virtuales SAABA-MEETING, con posibilidades de utilización de clases, seminarios, cursos, reuniones etc., en tiempo real, con una capacidad de 250 estudiantes divididos en cinco salas simultaneas, o en conferencias magistrales utilizando toda la capacidad en una sola sala.

\section{CONCLUSIÓNES}

a) Por la importancia que reviste el tema para el Instituto se ha ingresado como miembro en la REDDEES liderada por el CEPES de la Universidad de la Habana, como forma de intercambio de proyectos, de investigaciones así como del apoyo futuro que pudiéramos necesitar para la implementación de la modalidad en línea, exponiendo las primeras ideas en la reunión de la RED efectuada en Buenos Aires, Argentina..

b) Se ha trabajado en los aspectos teóricos metodológicos de la modalidad en línea y estamos en espera de los instructivos determinados para la normativa del Reglamento.

c) Contamos con la infraestructura tecnológica necesaria para el inicio de la modalidad en línea, al disponer de los servicios de la plataforma y aula virtual, necesarios según los estándares internacionales.

d) Queda establecido que la tendencia tanto nacional como en la provincia del Guayas, en lo relacionado con la acceso y conexión a internet, va en aumento año a año, y teniendo como apoyo las estrategias contenidas en el plan nacional de banda ancha del Ministerio de Telecomunicaciones y Sociedad de la Información, así como del plan "Guayaquil Ciudad Digital "del Muy Ilustre Municipalidad de Guayaquil.

e) De acuerdo a todo lo anterior se considera, que existen las condiciones mínimas para iniciar el proceso de implementación de la modalidad en línea en el Instituto, 


\section{BIBLIOGRAFÍA}

Alvarez R, Informe al Consejo Gubernativo sobre la Modalidad en línea en el AITEC. Vicerrectorado, (Marzo 2015) Agencia de Regulación y control de las telecomunicaciones. Información página WEB, 2015. Recuperado Junio de 2015

CES, Reglamento para Carreras y programas académicos en modalidades en línea, a distancia y Semipresencial o de convergencia de medios. Gaceta Oficial del CES. Diciembre de 2015.

INEC, Ecuador en cifras, Información página WEB -TICS (2013)

Meza,J, Modelo Pedagógico para proyectos de formación virtual. Ministerio Federal de la Cooperación Económica y Desarrollo. Bonn, Alemania. Mayo 2012

WIKIPEDIA, Educación en línea, http://es.wikipedia.org/Educación_en línea. Recuperado el 3 de Junio de 2015

Educacion $2.0 \mathrm{http} / / / \mathrm{www}$.educdoscero.com/2009/01/moodle-la-plataforma-Ims-libre-ms-usada.html. Recuperado el 18 de Marzo de 2016

\section{ANEXOS}

Situación de la cobertura de Internet a nivel nacional y en la provincia del Guayas.

Según el portal de Ecuador en cifras del Instituto Nacional de Estadísticas y Censos en su publicación acerca de las tecnologías de la información y comunicaciones (tic's) 2013, se pudieron recoger los siguientes datos:

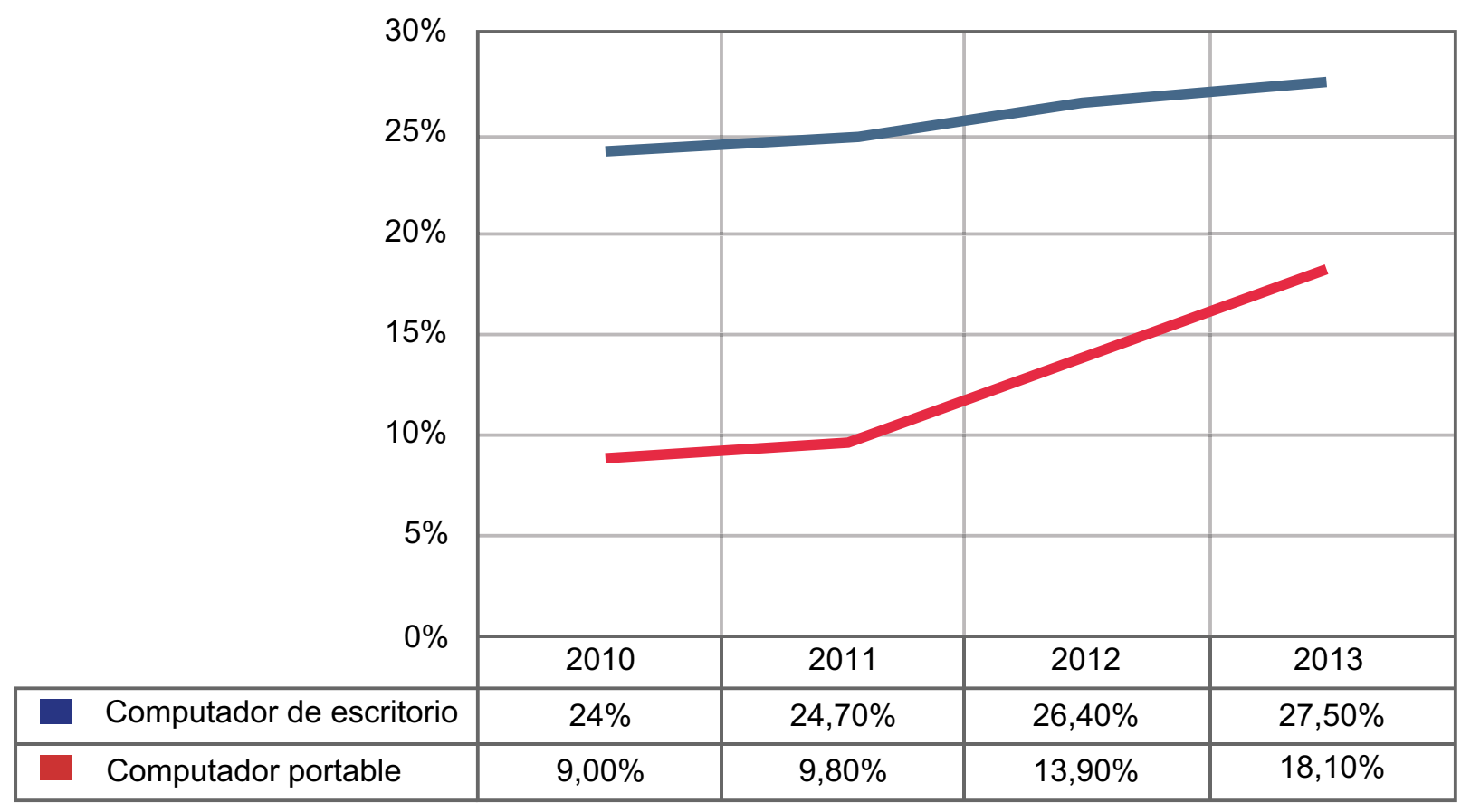

Figura 3 - El equipamiento tecnológico en el hogar a nivel nacional 


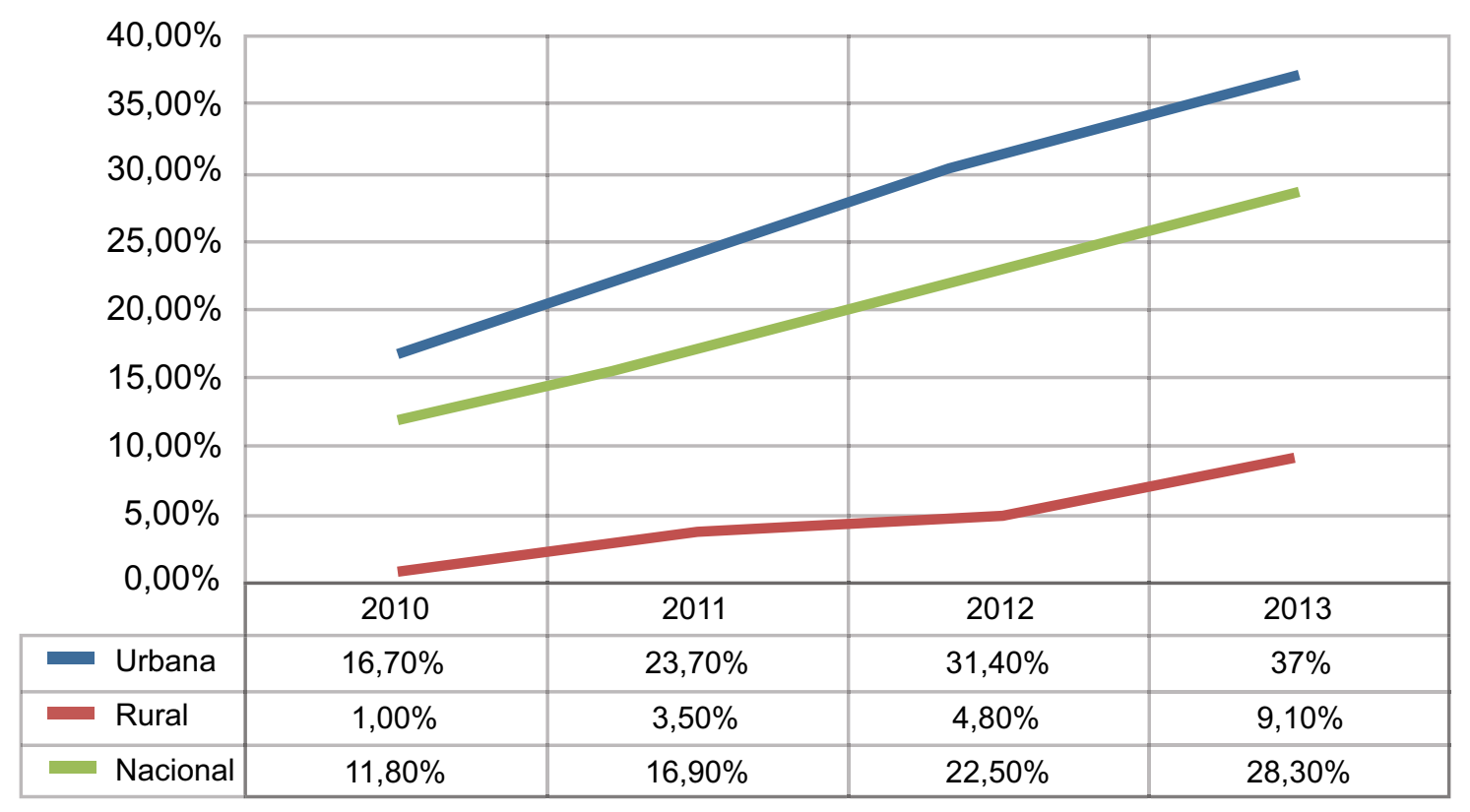

\section{Figura 4 - Acceso a internet de los hogares según área}

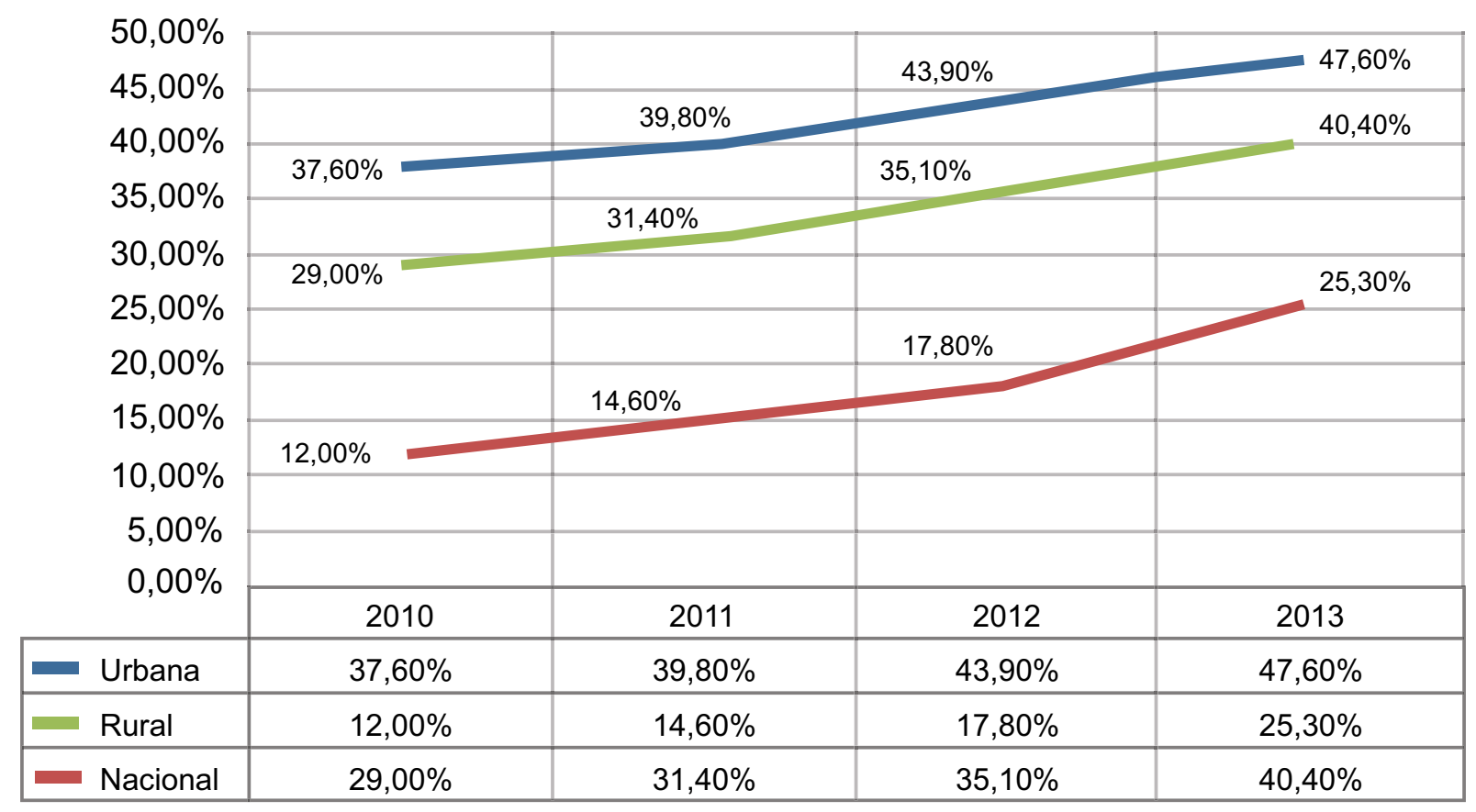

Figura 5 - Porcentaje de personas que han utilizado internet en los últimos 12 meses 


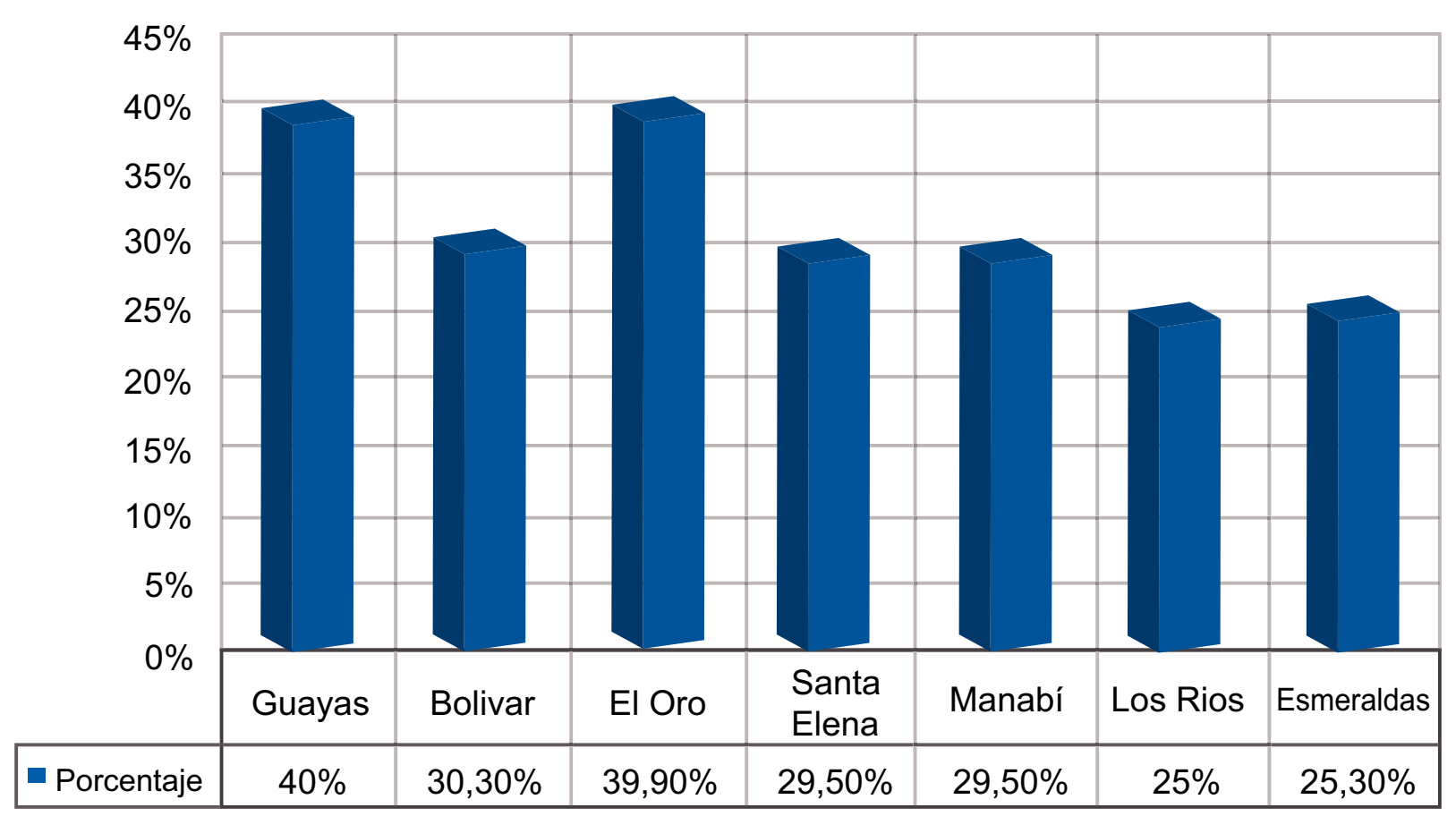

\section{Figura 6 - Porcentaje de personas que tienen acceso a internet}

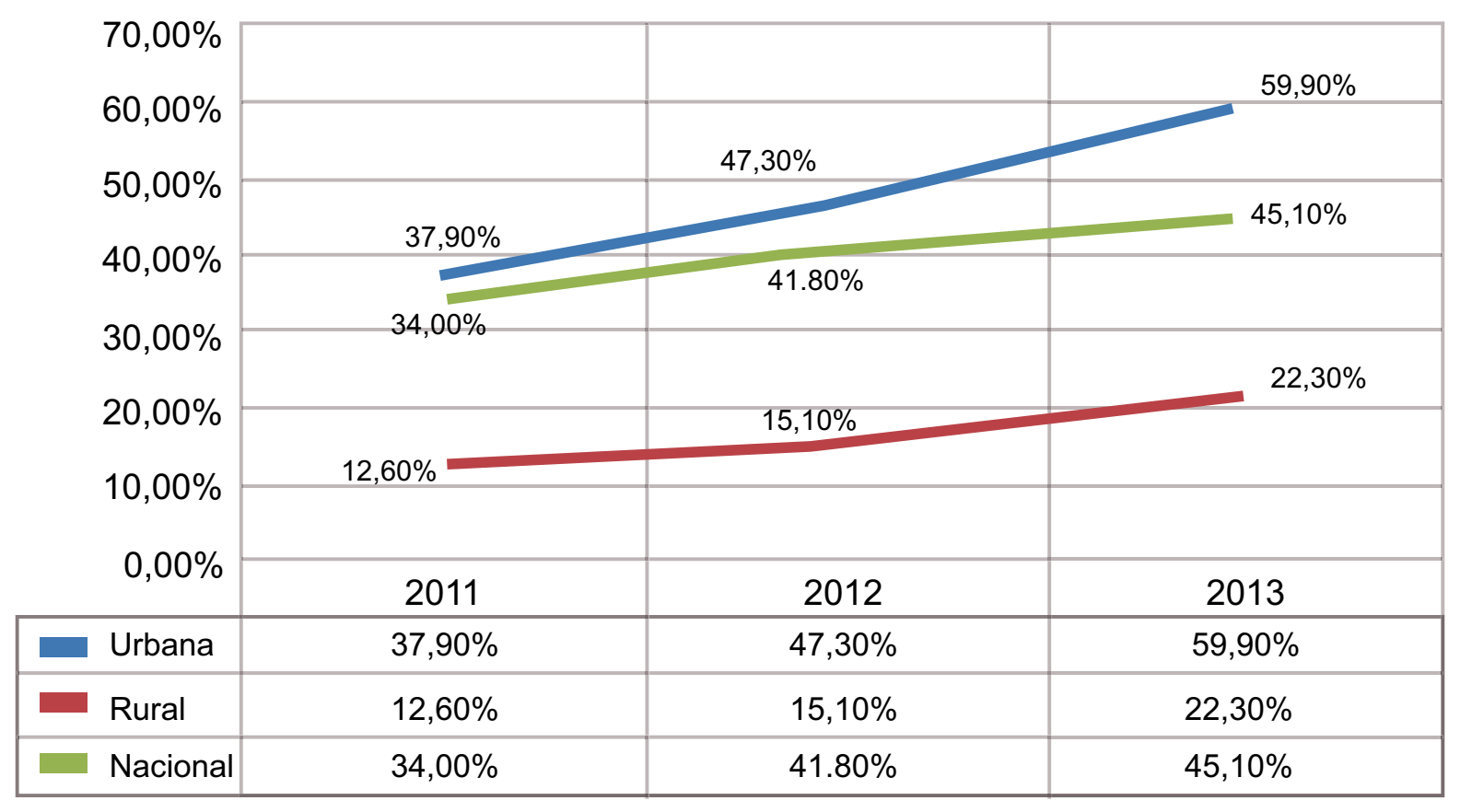

Figura 7 - Lugar de uso de internet por área en el hogar 
Figura 9 - Razones de uso de internet por área

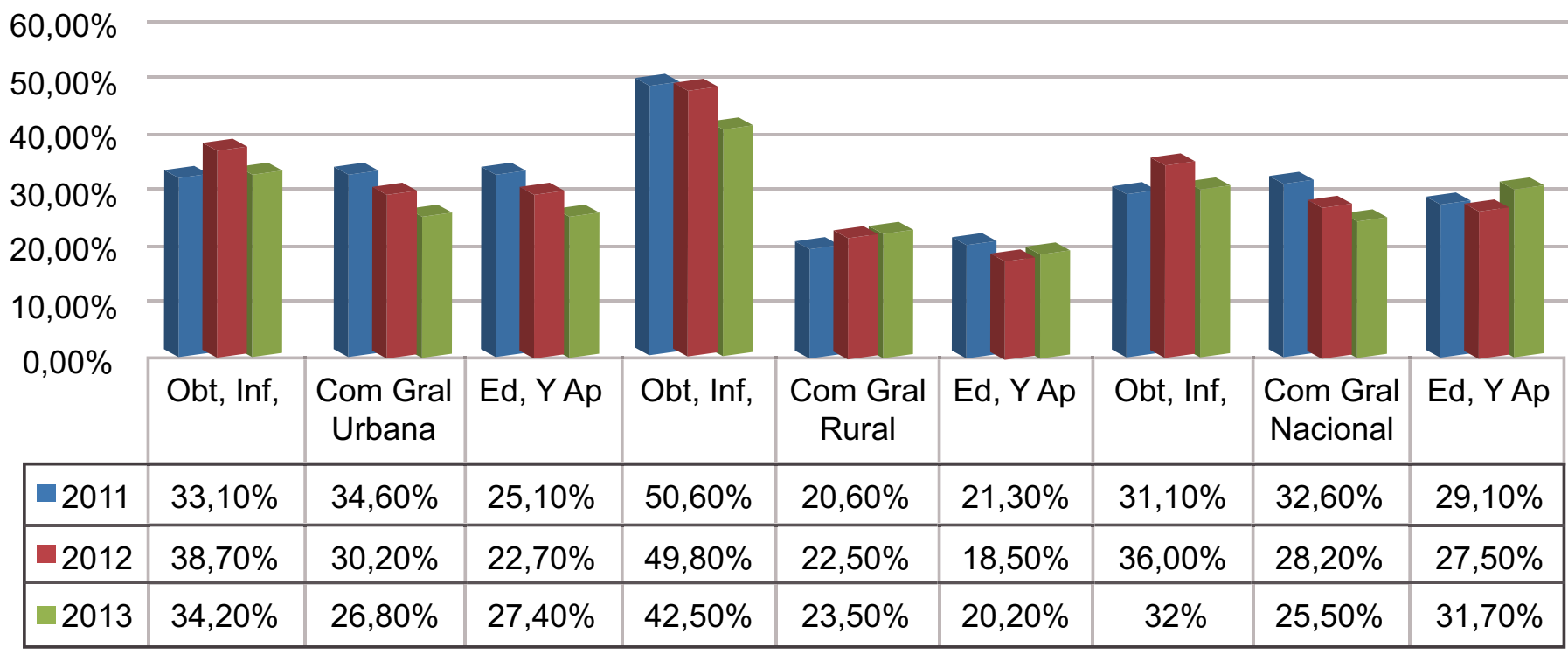

Fuente encuesta nacional de empleo desempleo y subempleo INEC,(2011-2013)

\begin{tabular}{|c|c|c|c|}
\hline & & & $\begin{array}{l}\text { lia de } \\
\text { ación y Control } \\
\text { Telecomunicaciones }\end{array}$ \\
\hline $\begin{array}{l}\text { Servicio d } \\
\text { Densidad de }\end{array}$ & $\begin{array}{l}\text { r Agregado de Int } \\
\text { (usuarios ) }\end{array}$ & & \\
\hline Fecha de publ & Julio 2015 & & \\
\hline AÑO & TOTAL DE USUARIOS & POBLACION & DESERDADAD \\
\hline 2001 & 249.021 & 12.479 .924 & $2,00 \%$ \\
\hline 2002 & 282.492 & 12.660 .728 & $2,23 \%$ \\
\hline 2003 & 364.153 & 12.842 .578 & $2,84 \%$ \\
\hline 2004 & 408.241 & 13.026.891 & $3,13 \%$ \\
\hline 2005 & 514.020 & 13.215 .089 & $3,89 \%$ \\
\hline 2006 & 823.483 & 13.408 .270 & $6,14 \%$ \\
\hline 2007 & 1.151 .906 & 13.605 .485 & $8,47 \%$ \\
\hline 2008 & 1.309 .605 & 13.805 .095 & $9,49 \%$ \\
\hline 2009 & 1.839 .634 & 14.005 .449 & $13,14 \%$ \\
\hline 2010 & 3.998 .362 & 14.483 .499 & $27,61 \%$ \\
\hline 2011 & 5.403 .833 & 14.765 .927 & $36,60 \%$ \\
\hline 2012 & 8.982 .014 & 15.520 .973 & $57,87 \%$ \\
\hline 2013 & 10.817 .644 & 15.774 .749 & $68,58 \%$ \\
\hline 2014 & 13.231 .169 & 16.027 .466 & $82,55 \%$ \\
\hline mar-15 & 14.043.191 & 16.090 .311 & $87,28 \%$ \\
\hline
\end{tabular}




\begin{tabular}{|c|c|c|c|c|c|}
\hline \multicolumn{6}{|c|}{$\begin{array}{l}\text { Servicio de Valor Agrega } \\
\text { Usuarios de Internet por } \\
\text { Acceso Fijo Marzo } 2015 \\
\text { Fecha de publicación: Julio } 2015\end{array}$} \\
\hline PROVINCIA & $\begin{array}{l}\text { USUARIOS } \\
\text { CONMUTADOS }\end{array}$ & $\begin{array}{l}\text { USUARIOS NO } \\
\text { CONMUTADOS } \\
\text { (DEDICADOS) }\end{array}$ & TOTAL & DENSIDAD & $\begin{array}{c}\text { POBLACIÓN } \\
\text { A MARZOO } \\
2015\end{array}$ \\
\hline AZUAY & 644 & 311287 & 311.931 & $39,00 \%$ & 799.730 \\
\hline BOLIVAR & 48 & 59544 & 59.592 & $29,78 \%$ & 200.118 \\
\hline CAÑAR & 0 & 85464 & 85.464 & $33,51 \%$ & 255.010 \\
\hline $\mathrm{CARCHI}$ & 52 & 54777 & 54.829 & $30,70 \%$ & 178.613 \\
\hline CHIMBORAZO & 124 & 257266 & 257.390 & $51,69 \%$ & 497.947 \\
\hline COTOPAXI & 296 & 153114 & 153.410 & $33,90 \%$ & 452.542 \\
\hline EL ORO & 1224 & 290722 & 291.946 & $43,90 \%$ & 664.958 \\
\hline ESMERALDAS & 136 & 136901 & 137.037 & $23,12 \%$ & 592.807 \\
\hline GALAPAGOS & 40 & 17486 & 17.526 & $60,63 \%$ & 28.908 \\
\hline GUAYAS & 1840 & 2355263 & 2.357 .103 & $58,34 \%$ & 4.040 .219 \\
\hline IMBABURA & 236 & 218150 & 218.386 & $49,58 \%$ & 440.445 \\
\hline LOJA & 3436 & 216918 & 220.354 & $44,84 \%$ & 491.395 \\
\hline LOS RIOS & 0 & 165732 & 165.732 & $19,35 \%$ & 856.552 \\
\hline MANABI & 368 & 442883 & 443.251 & $29,84 \%$ & 1.485 .547 \\
\hline $\begin{array}{l}\text { MORONA } \\
\text { SANTIAGO }\end{array}$ & 0 & 62870 & 62.870 & $36,59 \%$ & 171.810 \\
\hline NAPO & 20 & 62113 & 62.133 & $52,60 \%$ & 118.135 \\
\hline ORELLANA & 0 & 60325 & 60.325 & $40,44 \%$ & 149.174 \\
\hline PASTAZA & 12 & 62348 & 62.360 & $63,77 \%$ & 97.784 \\
\hline $\mathrm{PICHINCHA}$ & 2832 & 3082259 & 3.085 .091 & $106,18 \%$ & 2.905 .511 \\
\hline SANTA ELENA & 0 & 97327 & 97.327 & $27,60 \%$ & 352.692 \\
\hline $\begin{array}{l}\text { SANTO DOMINGO } \\
\text { DE LOS } \\
\text { TSACHILAS }\end{array}$ & 20 & 200243 & 200.263 & $48,49 \%$ & 412.996 \\
\hline SUCUMBIOS & 36 & 69205 & 69.241 & $34,30 \%$ & 201.889 \\
\hline TUNGURAHUA & 396 & 350621 & 351.017 & $63,53 \%$ & 552.515 \\
\hline $\begin{array}{l}\text { ZAMORA } \\
\text { CHINCHIPE }\end{array}$ & 240 & 40437 & 40.677 & $38,43 \%$ & 105.847 \\
\hline $\begin{array}{l}\text { ZONAS NO } \\
\text { DELIMITADAS }\end{array}$ & 0 & 124 & 124 & $0,33 \%$ & 37.171 \\
\hline TOTAL & 12.000 & 8.853 .379 & 8.865 .379 & $55,10 \%$ & 16.090 .311 \\
\hline
\end{tabular}

Tabla 9 Densidad de internet Acceso ffjo. 